\title{
EL INSTITUTO DE SEMIÓTICA LITERARIA, TEATRAL Y NUEVAS TECNOLOGÍAS DE LA UNED
}

\author{
José Romera Castillo \\ Universidad Nacional de Educación a Distancia
}

\section{HACIA UNA AUTOBIOGRAFÍA SEMIÓTICA}

La historia del Instituto está muy ligada a mis relaciones con la semiótica. Sus orígenes hay que buscarlos en mi quehacer docente e investigador, al que me referiré a continuación.

Al repasar mi trayectoria profesional, desde il mezzo del camin, me cabe la satisfacción de haber contribuido al estudio y fundamentación de los estudios semióticos en España. Desde que me doctoré en la Universidad de Granada, con una investigación sobre el Pluralismo crítico actual en el comentario de los textos literarios ${ }^{1}$ (1975), mis inclinaciones hacia la semiótica en general y, sobre todo, hacia la semiótica literaria y teatral en

\footnotetext{
${ }^{1}$ Granada: Universidad de Granada, 1976 (Resumen de Tesis de doctorado).
} 
particular han sido frecuentes como se puede comprobar tanto por mis trabajos - algunos de los cuales reseñaré después ${ }^{2}$ - como por la constatación que de los mismos han realizado los estudiosos del tema ${ }^{3}$.

Mi labor, como pionero e impulsor de la semiótica española, ha consistido, muy sintéticamente, en llevar a cabo varias actividades muy relacionadas entre sí.

${ }^{2}$ Cf. además Gramática textual. Aproximación semiológica a «Tiempo de silencio» (Valencia: Universidad, 1976); El comentario de textos semiológico (Madrid: Sociedad General Española de Librería, 1977; con $2{ }^{\text {a }}$ edición corregida y aumentada, El comentario semiótico de textos, 1980); Estudios sobre «El Conde Lucanor» (Madrid: UNED, 1980). Así como he editado el volumen La literatura como signo (Madrid: Playor, 1981). He publicado numerosos artículos sobre semiótica literaria: «Hacia una metodología estructuralista en el comentario de textos», Documenta (Valencia) XI (1974), 1-107 (mimeografiado); «Teoría y técnica del análisis narrativo», en J. Talens et alii, Elementos para una semiótica del texto artístico (Madrid: Cátedra, 1978, 111-152; con varias reediciones: $5 .^{\mathrm{a}}$ ed., 1995); «Función poética en Espadas como labios», Insula 374-75 (1978), 28 ( $\mathrm{N}^{\circ}$ extraordinario dedicado al poeta); «Juan Timoneda: Cómo comentar un texto en prosa: la estructura de un relato», en José Rico Verdú (ed.), Comentario de textos literarios (Madrid: UNED, 1980, 67-83) - sobre la patraña segunda-; «Selección bibliográfica sobre novela histórica», en J. Romera Castillo et alii (eds.), La novela histórica a finales del siglo XX (Madrid: Visor Libros, 1997, 427439); etc. Varios investigadores como, por ejemplo, Jesús L. Serrano Reyes, en su tesis de doctorado, luego publicada como libro, Didactismo y moralismo en Geoffrey Chaucer y don Juan Manuel: un estudio comparativo textual (Córdoba: Universidad, 1996), se basan, fundamentalmente, en mis propuestas metodologicas.

3 Cf. los trabajos, entre otros, de Ricardo Bellveser, «De Oscar Wilde a Juan GilAlbert. Con una referencia a José Romera», Las Provincias (Valencia), domingo 22 de febrero (1976), 40; las Comunicaciones presentadas en el Congreso 92 Annual Convention of Modern Language Association (Chicago, Illinois, 28 de diciembre, 1977) por el Equipo de la Revista Dieciocho, de Cornell University, «Current Semiotic Approaches to Hispanic Literary Criticism» y Cristina González, «Pilar Palomo, José Romera Castillo, Antonio García Berrio y Alicia Yllera. Los exponentes más recientes de la crítica semiológica española»; Adele L. Maragliano (1978), en su Memoria de Licenciatura, dirigida por Carolyn Richmond, presentada en el Departamento de Español de City University of New York, sobre el número monográfico de Ínsula (374$375,1974)$ dedicado a Vicente Aleixandre; Cristina González, «Los ejes MadridMálaga y Oviedo-Valencia: María del Pilar Palomo y José Romera Castillo», Dieciocho I.1 (1978), 77-81; Jesús M. ${ }^{\text {a }}$ Lasagabaster, «La crítica semiológica en España», Mundáiz 13 (1979), 7-21 [Apartado 2.2.3: «José Romera Castillo»]; Cristina González, «Semiotics in Spain», en T. A. Sebeok and J. Umiker Sebeok (eds.), The Semiotic Sphere (New York: Plenum Press, 1986, 478-481); Luis Alburquerque, «José Romera Castillo», en su artículo, «Producción bibliográfica de la teoría literaria española (I)», Revista de Literatura 109 (1993), 229-258 [especialmente 251-254]; Miguel Á. Garrido Gallardo, La Musa de la Retórica. Problemas y métodos de la ciencia de la literatura (Madrid: CSIC, 1994, 44, 48-49, 58, 279); María del Carmen Bobes Naves, «La semiología en España», en Isabel Paraíso (ed.), Retos actuales de la Teoría Literaria (Valladolid: Universidad, 1994, 11-30); Manuel Á. Vázquez Medel, «Nota preliminar», Discurso 9/10 (1996), 3; Tadeusz Kowzan, en «La semiologie du théâtre: ving-trois siècles ou vingt deux ans?», Diogène 149 (1990), 82-101 [Traducción: «¿La semiología del teatro: ¿veintitrés siglos o veintidós años?», en M. ${ }^{\mathrm{a}}$ del Carmen Bobes Naves (ed.), Teoría del teatro (Madrid: Arco Libros, 1997, 244)]; José Burgoa Abarca, 25 años de publicaciones periódicas de la UNED: indice bibliográfico de artículos (1972-1997) (Madrid: UNED, 1998, 240, 311 y 368); etc. 


\subsection{Creación de la Asociación Española de Semiótica y organización de varios Congresos}

Por iniciativa mía, el 23 de junio de 1983, creábamos la Asociación Española de Semiótica (AES) ${ }^{4}$, en el magno Congreso sobre Semiótica e Hispanismo, celebrado en Madrid - como se constata en los trabajos de José María Pozuelo Yvancos y Miguel Ángel Garrido en este número monográfico-, que se ha constituido, a lo largo de los años, en el núcleo aglutinador más importante de los estudiosos del tema en España, habiendo pertenecido a la Comisión gestora (1983-1984) y a la Junta Directiva como Secretario-Tesorero (1984-1988) y Presidente (1990-1994) por imperativo de sus estatutos.

La Asociación Española de Semiótica, desde 1984 y cada dos años, ha organizado ocho Congresos Internacionales importantísimos en Toledo, Oviedo ${ }^{5}$, Madrid, Sevilla, La Coruña, Murcia, Zaragoza y Granada, cuyas Actas - por el momento y hasta el VII Congresohan sido publicadas ${ }^{6}$. He tenido la oportunidad dichosa de participar muy directamente en varios de sus Simposia, al ejercer de director de la comisión local organizadora de dos de ellos: el I Simposio Internacional, sobre Teoría Semiótica ${ }^{7}$, celebrado en Toledo, del 7 al 9 de junio de 1984, y el III Simposio Internacional, sobre Retórica y Lenguajes, celebrado en la UNED, de Madrid, del 5 al 7 de diciembre de

4 Como figura en el libro de Actas de la Asociación. Tanto el Acta de fundación y los estatutos de la AES aparecen en los Apéndices I y IV de mi libro, Semiótica literaria y teatral en España (Kassel: Reichenberger, 1988, 167-168 y 180-189, respectivamente). Me he referido a la creación de AES en otros trabajos: «Semiótica literaria en España (Del letargo a la erupción)», en AES (ed.), Investigaciones Semióticas I (Actas del I Simposio Internacional) (Madrid: CSIC, 1986, 473-488); «La Asociación Española de Semiótica: información y balance», en $\mathrm{Da}$ Semiótica (Actas do I Colóquio Luso-Espanhol e do II Colóquio Luso-Brasileiro, Porto) (Lisboa: Vega / Universidade, 1988, 153-164); etc.

5 Las Actas de las Asambleas Generales de los dos Simposios Internacionales (de Toledo y Oviedo) aparecen publicadas en mi libro, Semiótica literaria y teatral en España (Kassel: Reichenberger, 1998, 169-173 y 174-179, respectivamente).

6 Investigaciones Semióticas I (Teoría Semiótica) (Sevilla / Madrid: CSIC, 1986); Investigaciones Semióticas II (Lo teatral y lo cotidiano) (Oviedo: Universidad, 1988, 2 vols.); Investigaciones Semióticas III (Retórica y Lenguajes) (Madrid: UNED, 1990, 2 vols.); Investigaciones Semióticas $N$ (Descubrir, inventar, transcribir el mundo) (Madrid: Visor, 1992, 2 vols.); Investigaciones Semióticas V (Semiótica y Modernidad) (La Coruña: Universidad, 1994, 2 vols.) e Investigaciones Semióticas VI (Mundos de ficción) (Murcia: Universidad, 1996, 2 vols.); Investigaciones Semióticas VII (Mitos) (Zaragoza: Anejos de Tropelías, 1998, 3 vols.). Las Actas del Congreso de Granada se publicarán próximamente.

7 Cf. José Romera Castillo, «I Simposio de la Asociación Española de Semiótica», A Distancia (UNED) 5 (1984), s. p. (Cuadernillo central). 
1988; así como he participado como editor de tres entregas de sus Actas: las del Congreso de Toledo, Investigaciones Semióticas I (Teoría Semiótica) (Sevilla / Madrid: Consejo Superior de Investigaciones Científicas, 1996); las del Simposio de Madrid, Investigaciones Semióticas III (Retórica y Lenguajes) (Madrid: UNED, 1990, 2 vols.; en colaboración con Alicia Yllera) ${ }^{8}$ y las del Congreso de Sevilla, Investigaciones Semióticas IV (Descubrir, inventar, transcribir el mundo) (Madrid: Visor Libros, 1992, 2 vols.) ${ }^{9}$. Además, siendo presidente de AES, creamos, en 1992, Signa. Revista de la Asociación Española de Semiótica, a la que me referiré después.

\subsection{AES y otras entidades semióticas}

Una vez constituida la Asociación Española de Semiótica, iniciamos el proceso de ponerla en relación con otros organismos nacionales e internacionales dedicados al estudio de la semiótica.

Por lo que concierne al ámbito español, constataré que siempre ha habido una excelente relación con otras entidades autonómicas dedicadas al estudio dicha esfera, como es el caso de la Asociación de Estudios Semióticos de Barcelona - ya desaparecida, dentro de la actividad semiótica en Cataluña, a la que se refieren Teresa Velázquez García-Talavera y Charo Lacalle Zaldueno en esta revista-, la Asociación Andaluza de Semiótica —de la que tratan aquí Manuel Ángel Vázquez Medel y Ángel Acosta-, la Asociación Vasca de Semiótica - de la que lamentablemente no tenemos un estudio en este número monográfico como hubiese sido mi deseo, pero que por razones evidentemente no semióticas el invitado que se comprometió a hacerlo nunca llegó a realizar el panorama solicitado-, la Asociación Gallega de Semiótica —estudiada aquí por José María Paz Gago y Pilar Couto Cantero- y el Centro de Semiótica y Teoría del Espectáculo, de la Universidad de Valencia, bajo la dirección de Jenaro Talens ${ }^{10}$, que también ha impulsado estos estudios. Con todos estos semilleros de inves-

8 Con reseña de Jesús G. Maestro, «La Retórica al día», Signa 1 (1992), 235-242.

9 Con «Presentación» de José Romera Castillo (t. I, 9) y reseña de Agustina Torres Lara, Epos (UNED, Madrid) IX (1993), 706-709.

10 Con la publicación, sobre todo, de los documentos de trabajo de Eutopías (2." época) con cerca de 100 folletos-volúmenes, publicados por Ediciones Episteme, cuyas contribuciones de tanto interés habrá que estudiar, en el futuro, con detenimiento. 
tigación he estado muy ligado, habiendo participado en la gestación de algunas asociaciones de semiótica como la Andaluza y la Gallega ${ }^{11}$.

$\mathrm{Y}$ por lo que respecta a organismos internacionales, AES ha estado muy ligada a la Association International for Semiotic Studies (AISS). En representación de España y de la AES, he sido fundador y Vocal de la Junta Directiva de la Federación Latinoamericana de Semiótica (desde 1987 hasta la actualidad) ${ }^{12}$ y presidente del IV Congreso de la citada Federación, que tendrá lugar en La Coruña en 1999; miembro del Comité Ejecutivo de la International Association for Semiotics Studies ${ }^{13}$ (1988-1993, elegido en Perpignan y reelegido en 1994 - hasta hoy-, en Berkeley) y miembro del Comité Directivo del International Semiotics Institute (1989-1994) ${ }^{14}$.

\subsection{Historia de la semiótica literaria y teatral en España}

Otro de los logros que me ha cabido llevar a cabo ha sido la realización de diversos panoramas de la evolución de la semiótica, fundamentalmente, en el ámbito de la teoría, la narratología, la poesía

11 Pertenezco como socio a la Asociación Andaluza de Semiótica, desde 1987 -habiendo sido Vocal de su Junta Directiva (1988-1992)_, así como a la Asociación Gallega de Semiótica, desde que se fundó.

12 Cf. la contribución de Lucrecia Escudero, «La Federación Latinoamericana de Semiótica ¿Existen los semiologos latinoamericanos?», en José Romera Castillo (ed.), «La semiótica en el ámbito hispánico (I)», Signa 7 (1998), 17-36.

13 Desde 1983 soy socio de la International Association for Semiotic Studies.

14 He tenido - y tengo- una participación muy activa en diversas publicaciones semióticas tanto en España como en el extranjero: Director de Signa. Revista de la Asociación Española de Semiótica (desde 1995) -como veremos luego-; Editor y del Editorial Committees de la revista S. European Journal for Semiotic Studies. Revue Européenne d'Études Sémiotiques. Europäische Zeitschrift für Semiotische Studien (Wien, Budapest, Madrid y Perpignan) — junto a Jeff Bernard (Editor-in-Chief), Gérard Deledalle, János Kelemen y Gloria Withalm—, desde el n. ${ }^{\circ}$ 7: 1,2 (1995); así como miembro del Consejo de Redacción de Signa y Discurso - de las que tratan Alicia Yllera y Ángel Acosta en este número-, Dieciocho. Hispanic Enlightenment Aesthetics and Literary Theory (Pennsylvania, Estados Unidos), Acta Poética (Universidad Autónoma de México), Semiosis (Universidad Veracruzana, Xalapa, México), Feuillets (Universidad de Fribourg, Suiza) —correspondant en España_, La Escena Latinoamericana (Instituto Internacional de Teoría y Crítica de Teatro Latinoamericano de Carleton University, Ottawa, Canadá), Anales de Literatura Española Contemporánea (University of Colorado at Boulder, USA -del Editorial Advisory Council: Drama / Theater-), etc.; y miembro de las Colecciones de publicaciones Problemata Semiotica y Teatro del Siglo de Oro (Kassel, Alemania: Editorial Reichenberger), Teoría y Crítica de la Cultura y Literatura (Frankfurt/Meim, Alemania: Editorial Vervuert Verlagsgesellschaft), etc. 
y el teatro en España hasta 1993, con una serie de trabajos que reseño a continuación: desde el libro editado en Alemania, Semiótica literaria y teatral en España ${ }^{15}$-y otros trabajos ${ }^{16}$ - hasta las diversas addendas ${ }^{17}$ que han ido completando el panorama. Por lo que se refiere a los trabajos sobre teatro en general ${ }^{18}$ y en español en particular (traducciones o investigaciones realizadas por hispa-

15 Kassel: Reichenberger, 1988. Con reseñas de Ángel García Galiano, Letras de Deusto 45 (1989), 206-207; Aida Amelia Porta, Incipit IX (1989), 235-236; Genara Pulido, Epos VI (1990), 595-597; Maria Grazia Profeti, Rassegna Iberistica 39 (1991), 33 y Floyd Merrell, «Un-Quixotic Semiotic?», Semiotica 86, 1-2 (1991).

16 Cf. los trabajos de José Romera Castillo, «La escuela valenciana de crítica literaria», Las Provincias (Valencia), 18 de junio (1978), VI; «Escorzo de la crítica semiótica española actual», Dieciocho. Hispanic Enlightenment Aesthetics and Literary Theory II.1 (1979), 3-20 - también en mi libro, El comentario semiótico de textos (Madrid: SGEL, 1980, 175-193, 2. ${ }^{2}$ ed.) -; «Panorama de la crítica semiótica de la literatura en España (1979-1983)», en Miguel Á. Garrido Gallardo (ed.), Teoría Semiótica. Lenguajes y textos hispánicos (Madrid: CSIC, 1985, 435-456); «Introducción a la semiótica (Notas bibliográficas)», en Antonio Sánchez Trigueros y José Valles Calatrava (eds.), Introducción a la Semiótica (Actas del Curso, Introducción a la Semiótica) (Almería: Instituto de Estudios Almerienses, 1992, 1-11); «La literatura medieval castellana desde la retina de la semiótica española», en María Isabel Toro Pascua (ed.), Actas del III Congreso de la Asociación Hispánica de Literatura Medieval (Salamanca: Biblioteca Española del siglo XV / Departamento de Literatura Española e Hispanoamericana, 1994, t. II, 893-903); «Panorama del análisis semiótico del cuento en España», en Peter Fröhlicher y Georges Güntert (eds.), Teoría $e$ interpretación del cuento (Berna: Peter Lang, 1995, 103-124; con 2.a ed. en 1996) - con reseña de Medardo Fraile, «Narratólogos y narradores», del volumen citado, en $S A B E R$ / Leer 95 (1996), 3-; etc. Cf. además los panoramas publicados en inglés, «Semiotics in Spain», en Thomas A. Sebeok and J. Umiker-Sebeok (eds.), The Semiotic Web '86. An International Yearbook (Berlín: Mouton de Gruyter, 1987, 291 348; en colaboración con Anna Carrascal) y «Literary semiotics in Spain: Bibliography», Semiotica 81, 3/4 (1990), 323-343.

17 Cf. José Romera Castillo, «Semiótica literaria y teatral en España. Addenda bibliográfica $1 \%$, en Roberto Pérez (ed.), Homenaje al profesor Ignacio Elizalde. Estudios literarios (Bilbao: Universidad de Deusto, 1989, 269-286) -el homenaje salió, con igual paginación y en el mismo año, en la revista Letras de Deusto 44-; «Semiotica literaria y teatral en España: addenda bibliográfica (I y II)», en José Romera y Alicia Yllera (eds.), Investigaciones Semióticas. III (Madrid: UNED, 1990, II, 537-561); «Semiótica literaria y teatral en España: addenda bibliográfica III», Discurso 6 (1991), 107-134; «Semiótica literaria y teatral en España: addenda bibliográfica IV», en AES (ed.), Investigaciones Semióticas. IV (Madrid: Visor Libros, 1992, II, 1.043-1.055); y «Semiótica literaria y teatral en España: addenda bibliográfica V», Signa 2 (1993), 167-184. Recogidas en mi libro, Literatura, teatro y semiótica: Método, prácticas y bibliografía (Madrid: UNED, 1998, 408-524).

18 Cf. José Romera Castillo, «Sobre texto y representación teatral», en Miguel Á. Muro (ed.), Actas del Congreso Internacional sobre: "Literatura Hispánica Actual. 1992" (Logroño: Gobierno de La Rioja / Consejería de Cultura, Deportes y Juventud, 1993, 291-311) - también como «Texto y representación desde la perspectiva semítica: un estado de la cuestión», en Alfredo Rodríguez López-Vázquez (ed.), Simposio "O Teatro e o seu ensino" (La Coruña: Universidade, 1993, 9-24); recogido en mi libro, Literatura, teatro y semiótica: Método, prácticas y bibliografía (Madrid: UNED, 1998, 193-225)- - y «El personaje en escena (Un método de estudio)», Jesús G. Maestro (ed.), Theatralia II. El personaje teatral (Vigo: Universidade, 1998, $77-$ 108). 
noamericanos), quedan constatados en otras entregas que complementan las anteriormente citadas ${ }^{19}$. El interesado en la historia de la semiótica literaria y teatral en España dispone con estos trabajos - unidos a los que otros investigadores han realizado sobre el tema- de un abundante banco de datos que radiografían lo que en el país de Cervantes se ha hecho desde la esfera semiótica en estos ámbitos.

\section{EL INSTITUTO DE SEMIÓTICA LITERARIA, TEATRAL Y NUEVAS TECNOLOGÍAS}

Además de lo anteriormente reseñado, hemos añadido un eslabón más a la cadena de las actividades semióticas en España. En 1989 pensé que era preciso crear un Centro Superior de Estudios Semióticos que sirviese para profundizar más en ellos y que, unido a lo ya existente y a lo que en otros lugares se estaba haciendo en el ámbito semiótico, sirviese como un punto de referencia más en el desarrollo de la misma. Y así nació bajo mi dirección, en 1991 -aunque pergeñado con anterioriedad- el Instituto de Semiótica Literaria y Teatral, inserto en el Departamento de Literatura Española y Teoría de la Literatura, de la Facultad de Filología de la Universidad Nacional de Educación a Distancia (UNED). Posteriormente - por el radio de acción de sus investigaciones - recibiría, desde 1996, el nombre que actualmente posee: Instituto de Semiótica Literaria, Teatral y Nuevas Tecnologías. El ISLTYNT —en el siglo de las siglas - ha pretendido conseguir varios objetivos, de los que ya ha producido granados frutos:

19 Cf. los panoramas de José Romera Castillo, «Semiótica teatral en España», en AES (ed.), Investigaciones Semióticas II (Actas del II Simposio Internacional) (Oviedo: Universidad, 1988, t. II, 353-388); «Semiótica teatral en español: ampliación bibliográfica», en José Romera y Alicia Yllera (eds.), Investigaciones Semióticas. III (Madrid: UNED, 1990, t. II, 563-571); «Crítica semiótica del teatro del Siglo de Oro en España», en Manuel García Martín (ed.), Estado actual de los estudios sobre el Siglo de Oro (Actas del II Congreso de AISO) (Salamanca: Universidad, 1992, t. II, 869-877). El trabajo «Sobre semiótica teatral en español (Una aproximación bibliográfica)», fue realizado para las Actas del Segundo Encuentro Internacional sobre Teatro Latinoamericano de hoy (Washington: The Catholic University of America), que hasta el momento no se han editado. 


\subsection{Seminarios Internacionales}

La primera actividad que quisiera destacar sería la llevada a cabo a través de la organización anual de un encuentro científico, de ámbito internacional, en el que destacados investigadores de España y del extranjero tuviesen la oportunidad de reunirse para exponer y discutir propuestas semióticas de trabajo sobre la literatura, el teatro y las nuevas tecnologías (puestas en relación con las áreas de conocimiento anteriormente mencionadas). Para ello, hemos elegido siempre un tema monográfico - y de actualidad-, que no hubiese sido estudiado en España con la profundización debida, con el fin de que prestigiosos investigadores, con invitación expresa, impartiesen las sesiones plenarias y los interesados en el tema pudiesen presentar comunicaciones, previamente seleccionadas antes de su exposición. A cada uno de los Seminarios Internacionales han asistido un grupo reducido de investigadores - no más de cincuenta-, con el fin de poder discutir amplia y profundamente los temas propuestos.

Son ocho los Seminarios Internacionales, celebrados hasta ahora, bajo mi dirección, cuyas Actas ha sido publicadas:

El primero, realizado en Segovia, del 3 al 5 de julio de 1991, se recogió en el número monográfico, que inició la andadura de una nueva revista, bajo la batuta de José Romera, Alicia Yllera y Rosa Calvet (eds.), Ch. S. Peirce y la literatura, Signa. Revista de la Asociación Española de Semiótica 1 (1992), 242 págs., con presentación de José Romera y Alicia Yllera (UNED), las sesiones plenarias de Dinda L. Gorlée (Research Center for Language and Semiotic Studies, Indiana University, Bloomington), M. ${ }^{\mathrm{a}}$ Lucía Santaella Braga (Pontifícia Universidade Católica de São Paulo), Floyd Merrell (Purdue University) y Robert Marty (Université de Perpignan), además de seis comunicaciones y tres estados de la cuestión, como se puede ver en el índice de Signa, de José Romera, que aparece en este número.

El segundo, celebrado en la sede de la UNED en Madrid, del 1 al 3 de julio de 1992, se publicó en José Romera, Alicia Yllera, Mario García-Page y Rosa Calvet (eds.), Escritura autobiográfica (Madrid: Visor Libros, 1993, 505 págs.) ${ }^{20}$, con presentación de José Romera (UNED), las sesiones plenarias de Darío Villanueva (Universidad de

20 Con reseña de Agustina Torres Lara, Signa 3 (1994), 285-289. 
Santiago de Compostela), Ángel G. Loureiro (University of Massachusetts, Amherst, Estados Unidos) y Francisco Hernández (Universidad de Valladolid), cuarenta y seis comunicaciones y un apéndice bibliográfico de José Romera Castillo (UNED).

El tercero, llevado a cabo en la capital de España, en colaboración con el Institut Français de Madrid, del 26 al 28 de abril de 1993, está compendiado en José Romera, Alicia Yllera y Mario García-Page (eds.), Semiótica(s). Homenaje a Greimas (Madrid: Visor Libros, 1994, 326 págs.) ${ }^{21}$, con presentación de José Romera (UNED), las sesiones plenarias de Estalisnao Ramón Trives (Universidad de Murcia), Eric Landowski (C.N.R.S. de París), Laimonas Tapinas -entrevista-, Denis Bertrand (BELC-CIEP de París), Eduardo Peñuela Cañizal (Universidade de São Paulo), Jean-Marie Floch (Institut d'Études Politiques de París), José María Nadal (Universidad de El País Vasco), Helena Usandizaga (Universidad Autónoma de Barcelona) y Fernando Poyatos (University of New Brunswick, Canadá) —además de Jorge Lozano (Universidad Complutense de Madrid), cuyo trabajo no aparece en las Actas por voluntad expresa del autor-, así como diecienueve comunicaciones.

El cuarto, que tuvo lugar en la sede de la UNED de Madrid, del 4 al 6 de julio de 1994, está publicado en José Romera Castillo, Mario García-Page y Francisco Gutiérrez Carbajo (eds.), Bajtín y la literatura (Madrid: Visor Libros, 1995, 459 págs.) ${ }^{22}$, con presentación de José Romera (UNED), las sesiones plenarias de Iris M. Zavala (Universidad de Utrech, Holanda) y Augusto Ponzio (Università de Bari, Italia), treinta y cuatro comunicaciones y un apéndice bibliográfico de José Romera Castillo (UNED).

El quinto, celebrado en la sede de la Universidad Internacional Menéndez Pelayo de Cuenca, del 3 al 6 de julio de 1995, puede leerse en José Romera Castillo, Francisco Gutiérrez Carbajo y Mario GarcíaPage (eds.), La novela histórica a finales del siglo XX (Madrid: Visor Libros, 1996, 439 págs.) ${ }^{23}$, con presentación de José Romera (UNED), las sesiones plenarias de Maryse Bertrand de Muñoz (Uni-

21 Con reseña de Emilia Cortés Ibáñez, Signa 4 (1995), 265-269.

22 Con reseñas de Darío Villanueva, $A B C$ Cultural 201, 8 de septiembre (1995), 13; Laura Serrano de Santos, Signa 5 (1996), 361-364 y José Manuel Pérez Carrera, Epos XII (1996), 626-628.

${ }^{23}$ Con reseñas de José Enrique Martínez, Suplemento cultural de El Mundo, octubre (1996); Emilia Cortés Ibáñez, Signa 6 (1997), 439-445 y Alfredo Rodríguez López-Vázquez, Lenguaje y Textos (La Coruña) 10 (1997), 375-376. 
versité de Montreal, Canadá), M. ${ }^{\text {a }}$ del Carmen Bobes (Universidad de Oviedo), Carlos García Gual (Universidad Complutense de Madrid), Germán Gullón (Universitëit van Amsterdam, Holanda), Miguel Herráez (Universidad de Valencia), Joan Oleza (Universitat de València) y José M. ${ }^{\text {a }}$ Pozuelo Yvancos (Universidad de Murcia), treinta y cuatro comunicaciones y dos apéndices bibliográficos de Alfredo Caunedo (Grupo de Investigación del ISLTYNT) y José Romera Castillo (UNED).

El sexto, que se realizó en la Universidad Internacional Menéndez Pelayo de Cuenca, del 1 al 4 de julio de 1996, fue publicado por José Romera Castillo, Francisco Gutiérrez Carbajo y Mario García-Page (eds.), Literatura y multimedia (Madrid: Visor Libros, 1997, 386 págs.) ${ }^{24}$, con presentación de José Romera (UNED), las sesiones plenarias de José Romera Castillo (UNED), Antonio R. de las Heras (Universidad Carlos III de Madrid), Francisco A. Marcos Marín (Universidad Autónoma de Madrid), Germán Ruipérez (UNED), Enric Bou (Brown University, Estados Unidos), Joaquín M. a Aguirre (Universidad Complutense de Madrid) y Francisco Gutiérrez Carbajo (UNED) —además de las de Jenaro Talens (Universidad de Valencia), Javier Blasco (Universidad de Valladolid), Orlando Carreño y Jorge Urrutia (Universidad Carlos III) que no aparecieron publicadas por voluntad expresa de sus autores-, trece comunicaciones sobre «Literatura y multimedia»y otras seis sobre «Enseñanza de la literatura».

El séptimo, que tuvo lugar en la Casa de Velázquez de Madrid, del 26 al 29 de mayo de 1997, ha aparecido en José Romera Castillo y Francisco Gutiérrez Carbajo (eds.), Biografías literarias (1975-1997) (Madrid: Visor Libros, 1998, 647 págs.), con presentación de José Romera (UNED), las sesiones plenarias de Ricardo Senabre (Universidad de Salamanca), Daniel Madelénat (Université Blaise-Pascal, Francia), Miguel Ángel Pérez Priego (UNED), José Montero Reguera (Universidade de Vigo), Francisco Aguilar Piñal (CSIC), Leonardo Romero Tobar (Universidad de Zaragoza), Rafael Alarcón Sierra (Universidad de Zaragoza), Andrés Soria Olmedo (Universidad de Granada), José Romera Castillo (UNED) y Marcos Ricardo Barnatán (Escritor y crítico) —además de la intervención del escritor Juan Manuel de Prada

24 Con reseñas de Mar Cruz Piñol, Signa (1998) 7, 397-403 —en versión impresa-y Espéculo 6, julio (1997): (http://www.ucm.es/OTROS/especulo/numero6/lit_mult.htm) -en formato electrónico- y José Antonio Millán, «Los nuevos soportes del texto», El País (Babelia 360), 3 de octubre (1998), 13. 
que no aparece publicada por voluntad expresa de su autor- y treinta y dos comunicaciones.

El octavo, que se llevó a cabo en la Universidad Internacional Menéndez Pelayo de Cuenca, del 25 al 28 de junio de 1998, podrá leerse en José Romera Castillo y Francisco Gutiérrez Carbajo (eds.), Teatro histórico (1975-1998): textos y representaciones (Madrid: Visor Libros, 1999, en prensa), con presentación de José Romera (UNED), las sesiones plenarias de José Romera Castillo (UNED), César Oliva (Universidad de Murcia), Juan Villegas (University of California, Irvine, Estados Unidos), Ángel Berenguer (Universidad de Alcalá), Beatriz Hernanz (crítica), Josep Lluis Sirera (Universitat de València), Mariano de Paco (Universidad de Murcia), Virtudes Serrano (Universidad de Murcia), Juan A. Ríos Carratalá (Universidad de Alicante), Nel Diago (Universitat de València), María Francisca Vilches de Frutos (CSIC), María-José Ragué (Universitat de Barcelona), Francisco Gutiérrez Carbajo (UNED) y los dramaturgos Eduardo Galán - Subdirector General de Teatro-y José María Rodríguez Méndez, además de treinta y siete comunicaciones.

El noveno, tendrá lugar en la UNED (Madrid), del 21 al 23 de junio de 1999, sobre Poesía histórica y (auto)biográfica (1975-1999).

Todo un ramillete de estudios que servirán de guía a los investigadores de la semiótica interesados en los temas propuestos. Puedo afirmar, con gran complacencia —como vengo sosteniendo-, que nuestros Seminarios Internacionales ocupan un lugar destacado -creoen el panorama de la investigación semiótica tanto en España como fuera de ella, como ha sido reconocido por la crítica.

\subsection{Signa. Revista de la Asociación Española de Semiótica}

Desde que creara la Asociación Española de Semiótica siempre tuve en mente la creación de una revista que sirviese para dar cauce a investigaciones semióticas tanto de España como del extranjero. Con tal fin, en 1992, siendo Presidente de AES, impulsé la creación de Signa. Revista de la Asociación Española de Semiótica, habiéndose publicado, hasta el momento, ocho números. El n. ${ }^{\circ} 1$ (1992) de la revista, de carácter monográfico, recogió las Actas del I Seminario Internacional de nuestro Instituto, sobre Charles Sanders Peirce y la literatura, 
como hemos visto. La dirección de los tres primeros números ${ }^{25}$ la llevó a cabo la destacada semiótica Alicia Yllera, correspondiéndome a mí los cinco siguientes ${ }^{26}$. En consecuencia, siempre he estado muy vinculado a ella al haber pertenecido a su Consejo de Redacción. De ello trata Alicia Yllera en un trabajo en este número de la revista, al que remito.

\subsection{Investigaciones semióticas}

Son varias las líneas de investigación seguidas en el Instituto, bajo mi dirección y hasta el momento:

\subsubsection{Semiótica teatral: reconstrucción de la vida escénica}

El profesor universitario, además de cumplir con sus deberes docentes, tiene una de las labores más estimulantes - y más gozosas-, cual es promover la investigación entre sus discípulos. Puedo decir, si me permite, sin sonrojo que estoy enormemente satisfecho de haber contribuido - no sé con qué tino: el tiempo se encargará del dictamena este objetivo. Desde mis años de formación en la Universidad de Granada - a la que tanto debo-, por experiencia propia, y posteriormente a través de mi actuación universitaria, se ha ido afianzando en mí la idea de que la investigación en general - y la teatral en particular- es mucho más productiva, a la larga, si se realiza en equipo, con proyectos bien diseñados y objetivos claramente marcados.

Cualquier investigador del teatro español sabe que los estudios sobre la historia del texto dramático son abundantes; pero también sabe que los referidos a la representación, a la puesta en escena, en un orden diacrónico, son cuánticamente menos numerosos, aunque, es cierto, que en los últimos tiempos el panorama está cambiando. En suma, conocemos muy bien la historia literaria de los autores y de los textos

\footnotetext{
251 (1992), 2 (1993) y 3 (1994).

264 (1994), 5 (1996), 6 (1997), 7 (1998) y 8 (1999).
} 
dramáticos; pero sabemos mucho menos de la historia de las obras que fueron contempladas y degustadas por nuestros antecesores.

Para subsanar esta laguna - a veces océano inmenso- se han llevado a cabo - y se siguen haciendo- una serie de actividades dignas del mayor encomio. Por lo tanto, nuestros trabajos - ni que decir tiene- se engarzan con dichas investigaciones tanto individuales - que no puedo pormenorizar aquí- como de grupo (por ejemplo, las del CSIC, bajo la dirección de María Francisca Vilches de Frutos y Dru Dougherty ${ }^{27}$; las de la Universidad de Alcalá, bajo la dirección de Ángel Berenguer ${ }^{28}$, sobre el teatro representado en Madrid; las de la Universidad de Alicante, centradas en la actividad escénica de la ciudad levantina en la segunda mitad del siglo XX, bajo la dirección de Juan Antonio Ríos Carratalá ${ }^{29}$; etc.) que tanta luz van aportando a la historia del teatro representado en España ${ }^{30}$. A los autores de todas ellas, en general y desde estas líneas, quisiera agradecer el ingente trabajo realizado.

Nuestras aportaciones se caracterizan por estar insertas en un amplio grupo de trabajo, diseñado con rigor y minuciosidad, sobre la semiosis de todos los componentes que articulan la representación en diferentes lugares de España y del extranjero, preferentemente durante los siglos XIX (segunda mitad) ${ }^{31}$ y siglo XX. Las características de mi alumnado de la UNED así lo determinaban. El interés del trabajo de investigación ha sido ya reconocido al ser subvencionado por la

27 Cf. Dru Dougherty y María Francisca Vilches de Frutos, La escena madrileña entre 1918 y 1926. Análisis y documentación (Madrid: Fundamentos, 1990) y María F. Vilches y Dru Dougherty, La escena madrileña entre 1926 y 1931. Un lustro de transición (Madrid: Fundamentos, 1997). Además de las carteleras teatrales españolas de los últimos años, publicadas por M. ${ }^{\mathbf{a}}$ Francisca Vilches de Frutos, La temporada teatral española 1982-1983 (Madrid: CSIC, 1983) o en diversas entregas en la revista Anales de la Literatura Española Contemporánea; así como los numerosos trabajos publicados por diversos componentes del grupo, tesis de doctorado, etc.

${ }_{28} \mathrm{Cf}$. por ejemplo Manuel Pérez, La escena madrileña en la transición política (1975-1982), Teatro. Revista de Estudios Teatrales 3-4 (1993) y Juan Pedro Sánchez Sánchez, La escena madrileña entre 1970 y 1974, Teatro. Revista de Estudios Teatrales 12 (1997), 390 págs. (números monográficos de la citada revista).

29 Como por ejemplo la tesis de doctorado de Eva García Ferrón, El teatro en Alicante entre 1966 y 1993 (Alicante: Universidad, 1997; versión electrónica, 2 disquetes).

30 En el Departamento de Historia del Arte, de la UNED, en 1997, se defendió la tesis de doctorado de Juana María Balsalobre García, Imagen académica del teatro español decimonónico. El teatro y su censura en la Real Academia de Bellas Artes de San Fernando (1800-1870), bajo la dirección del Dr. José Enrique García Melero.

31 Cf. José Romera Castillo, «Teatro regional español en el siglo XIX (Bibliografía)», en José Romera, Antonio Lorente y Ana M.a Freire (eds.), Ex Libris. Homenaje al profesor José Fradejas Lebrero (Madrid: UNED, 1993, t. II, 705-718). 
Dirección General de Investigación Científica y Técnica (DGICYT) y la Dirección General de Enseñanza Superior del Ministerio de Educación y Cultura ${ }^{32}$, así como por los estudiosos del tema ${ }^{33}$.

Nuestro trabajo consiste, muy sintéticamente, en lo siguiente. Una vez elegido el punto geográfico en el que se investigará la vida escénica en toda su amplitud en un periodo determinado de años -en función de la documentación que sea accesible-, se traza, en primer lugar, un panorama histórico, literario y teatral de la época tanto general de España como de la ciudad en particular. A continuación se compila un estado de la cuestión sobre la bibliografía referida a la actividad escénica en el lugar seleccionado, estableciendo las fuentes documentales (archivos, hemerotecas, etc.) sobre las que se basará la investigación. Después se estudian los espacios teatrales de la localidad (teatros propiamente dichos y otros ámbitos como Casinos, Ateneos, casas particulares, etc.).

Asentadas las bases de la investigación, entramos de lleno en el núcleo fundamental de la misma, constituido por el establecimiento de la cartelera teatral (obras representadas y obras anunciadas que no llegaron a ponerse en escena por diversos motivos), desde un punto de vista cronológico, en la que se constatan para cada año, mes, día y hora, por funciones, en las sesiones de tarde y noche, los títulos de las piezas y los nombres de los autores que se representaron, los lugares escénicos, así como las compañías y actores, consignando siempre la referencia bibliográfica de donde se toman los datos.

Una vez establecida la cartelera, nos centramos en la relación de las obras (con un índice alfabético y una relación númerica de las veces que las obras se pusieron en escena) y su clasificación por géneros (comedias, tragedias, dramas, melodramas, etc.) tanto del teatro declamado (obras originales o traducciones-adaptaciones de piezas de teatro foráneo - la mayoría de las veces francesas-), como del teatro lírico musical (ópera, zarzuela) y género chico, Intermedios (bailes, canciones, poesías, etc.) y espectáculos parateatrales (acrobacias, malabarismos, magias, etc.). Después se estudian los autores (dramaturgos, adaptadores, libretistas y compositores), con sus obras respectivas.

32 Proyectos PS90-0104 (1990-1993) y PB96-0002 (1997-2000), respectivamente.

33 Cf., por ejemplo, César A. Archaga Martínez, Actividades dramáticas en el Teatro Principal de Burgos: 1858-1946 (Burgos: Ayuntamiento, 1997, 21); etc. 
A continuación, nos centramos en todo lo relacionado con la representación teatral en su conjunto. En primer lugar, atendemos a las compañías (tanto profesionales como de aficionados -que tanto proliferaron-), estableciendo la relación de sus componentes y el repertorio de obras representadas. Como consecuencia de este estudio, tenemos ya unos itinerarios de las diversas compañías que recorrieron todo el territorio nacional. Después, pasamos al estudio de los distintos lenguajes escénicos (escenografía, música, vestuario, luminotecnia, etc.). Para ello nos servimos del marco teórico de la semiótica teatral que tanto ha aportado al respecto. Los resultados de este apartado son de extraordinario valor para la historia de la representación escénica, sobre la que poseemos escasos datos, ya que desafortunadamente no existían los medios tecnológicos de hoy (grabaciones de televisión, vídeo, etc.), y que gracias a estas aportaciones vamos teniendo noticias más pormenorizadas.

Después analizamos la recepción crítica de los espectáculos teatrales, examinando la relación de críticos (siempre que es posible) así como la crítica de los autores, de los textos dramáticos, de las compañías, de las puestas en escena, etc.

La sociología del hecho teatral no podía estar ausente. Desde las reglamentaciones oficiales sobre el teatro (tanto nacionales como provinciales), problemas de censura, representaciones a beneficio (de Instituciones, actores, etc.), aficiones poéticas y musicales en los intermedios, regalos, homenajes y agradecimientos, etc. Después - hecho importantísimo-, estudiamos las temporadas teatrales (épocas y meses del año, días de la semana, horario de las funciones, etc.); analizamos los precios (por abonos y entradas sueltas); para terminar recogiendo las costumbres del público asistente a las representaciones que eran muy pintorescas en ocasiones (sombreros de las señoras que no dejaban ver bien el escenario, comportamientos soeces, etc.).

Teniendo como colofón unas conclusiones generales, con un análisis de la vida escénica en el lugar escogido, y que, por sí mismas, constituyen una pequeña monografía lista para la imprenta; además de las referencias bibliográficas y una serie de apéndices, sobre todo gráficos (planos de teatros, fotografías, documentos manuscritos, programas de mano, anuncios en periódicos, etc.), que hasta el momento eran, en general, desconocidos.

$\mathrm{Y}$ es todo. Como se puede colegir, un amplio abanico que trata de reconstruir muy pormenorizadamente no sólo las informaciones que las 
fuentes documentales aportan, día tras día, sino que pretenden realizar, sobre todo, un análisis valorativo y comparativo con lo ya conocido de las actividades escénicas en su integridad del punto geográfico elegido.

En estos catorce años de vida del proyecto, desde que 1984 empezamos a trabajar, hemos producido enjundiosos frutos (tesis de doctorado y Memorias de Investigación), como a continuación enumero.

Sobre la segunda mitad del siglo XIX se ha investigado la vida escénica, a través de tesis de doctorado, en siete ciudades. He aquí la relación: Emilia Cortés Ibáñez, El teatro en Albacete en la segunda mitad del siglo XIX (1991) — publicada en microforma por la UNED en 1991 - ; José Antonio Bernaldo de Quirós, El teatro y actividades afines en Ávila (siglos XVII, XVIII y XIX) (1993) -publicada en microforma por la UNED en 1994 y posteriomente como Teatro y actividades afines en la ciudad de Ávila (Siglos XVII, XVIII y XIX) (Ávila: Diputación Provincial / Institución Gran Duque de Alba, 1998, 342 págs.)_; Ángel Suárez Muñoz, La vida escénica en Badajoz 18601886 (1994) - publicada en microforma por la UNED en 1995 y posteriormente como El teatro en Badajoz: 1860-1886. Cartelera y estudio (Madrid: Támesis, 1997, 343 págs., Colección «Fuentes para la historia del teatro en España», n. ${ }^{\circ}$ XXVIII)-; María del Mar López Cabrera, El teatro en Las Palmas de Gran Canaria (1853-1900) (1995) - publicada en microforma por la UNED en 1995-; Agustina Torres Lara, La escena toledana en la segunda mitad del siglo XIX (1996); Tomás Ruibal Outes, La vida escénica en Pontevedra en la segunda mitad del siglo XIX (1997) — publicada en microforma por la UNED en 1998- y Estefanía Fernández García, León y su actividad escénica en la segunda mitad del siglo XIX (1997) —publicada en microforma por la UNED en 1998-.

Además se han realizado siete Memorias de Investigación -inéditas- sobre algunos años concretos, que, en el futuro, se convertirán algunas de ellas en tesis de doctorado: El teatro en Cádiz (1867-1870), de Amalia Vilches Dueñas (1984); El teatro en Calahorra (18401910), de María Á. Martínez Somalo (1988); El teatro en Córdoba (1854-1858), de María Teresa Gómez Borrego (1988); El teatro en Barcelona en la segunda mitad del siglo XIX (1850-1854), de Ana María Grau Gutiérrez (1992); El teatro en Igualada en la segunda mitad del siglo XIX (1863-1880), de María Rosa Vila Farré (1992); El teatro en Bilbao (1890-1892), de Begoña Alonso Bocos (1996) y La vida escénica en el teatro Jofre de Ferrol: 1892-1896, de M. ${ }^{a}$ Eva Ocampo Vigo (1997). 
Sobre el siglo XX, se han defendido tres tesis de doctorado. He aquí su relación: Francisco Reus-Boyd-Swan, El teatro en Alicante (19001910) (1991) — publicada, primeramente, en microforma por la UNED en 1992 y posteriormente como El teatro en Alicante: 1901 1910. Cartelera y estudio (Madrid / Londres: Támesis / Generalitat Valenciana, 1994, 438 págs., Colección «Fuentes para la historia del teatro en España», n. ${ }^{\circ} \mathrm{XXIII)-;} \mathrm{Francisco} \mathrm{Linares} \mathrm{Valcárcel,} \mathrm{La} \mathrm{vida}$ escénica en Albacete (1901-1923) (1997) —publicada en microforma por la UNED en 1998- y Emilia Ochando Madrigal, El teatro en Albacete (1924-1936) (1998) - publicada en microforma por la UNED en 1998-. Asimismo, Paloma González-Blanch Roca, ha realizado su Memoria de Investigación sobre La vida escénica en Segovia (1918-1923) (1998). Hay otras en curso de realización ${ }^{34}$.

Hay que decir que las diez tesis de doctorado han sido publicadas -o aguardan su publicación- en microforma por la UNED. Algunas de ellas, además, han sido editadas por la prestigiosa Colección «Fuentes para la historia del teatro en España», de la editorial londinense / madrileña Tamesis Books (Badajoz y Alicante), por Instituciones locales (Ávila) y algunas otras serán publicadas pronto por entidades provinciales como la Diputación de Albacete, etc.

En conjunto, por lo que respecta al siglo XIX, se han investigado catorce puntos geográficos de España: Albacete, Ávila -que cubre los siglos XVII, XVIII y XIX - , Badajoz, Las Palmas de Gran Canaria, Toledo, Pontevedra y León - sobre la vida escénica en la segunda mitad del siglo XIX-; además de algunos años de las carteleras teatrales de Cádiz, Calahorra, Córdoba, Barcelona, Igualada, Bilbao y Ferrol.

Por lo que concierne al siglo XX, se han realizado, bajo mi dirección, las carteleras de Alicante (1900-1910), Albacete (1901-1936) y Segovia (1918-1923); así como algunas Memorias sobre algunas temporadas teatrales, integradas en el equipo de investigación, realizadas

34 Añadiré que se han realizado Memorias de Investigación, integradas en nuestro Instituto, sobre temporadas teatrales en otros puntos de la geografía española, bajo la tutela de mis colaboradores, Pilar Espín Templado: El teatro en La Rioja en la segunda mitad del siglo XIX, de Inmaculada Benito Argáiz (1997) -defendida en la Universidad de La Rioja en 1997 (codirigida por Miguel Ángel Muro)_; El teatro en Badajoz en la primera mitad del siglo XX (1900-1902), de Pablo Fernández García (1995) y La vida escénica en Pontevedra en la primera mitad del siglo XX (19011903), de Paulino Aparicio Moreno (1996); así como de Francisco Gutiérrez Carbajo: Representaciones teatrales y compañías de teatro profesional gallegas en 1993, de Fernando Dacosta Pérez (1998). Además de otras en preparación. 
bajo la dirección de mis colaboradores Pilar Espín Templado (La Rioja, Badajoz y Pontevedra) y Francisco Gutiérrez Carbajo (Galicia).

Pero el ámbito de actuación de nuestro equipo no se detiene sólo en España, sino que están terminándose dos investigaciones (tesis de doctorado) sobre La actividad escénica en Guadalajara (México) 19201990, de Alfredo Cerda Muños y La vida escénica del teatro español del siglo XX en Italia, de Coral García Rodríguez.

En síntesis, las pesquisas en cerca de una veintena de puntos geográficos de España - entre los que resaltan los amplios panoramas de Albacete y Ávila_, más las dos del extranjero (México e Italia), constituyen un corpus muy significativo que arroja una intensísima luz no sólo de la vida escénica de estos lugares, sino que, gracias a nuestra iniciativa y sobre todo al esfuerzo de nuestros investigadores - a los que desde aquí quiero reconocer y agradecer su denodado esfuerzo-, al estar ya cruzando y comparando los datos, la historia de nuestro teatro representado ya no es una anhelada utopía, sino una rigurosa y bien fundada realidad.

\subsubsection{Escritura autobiográfica}

El segundo núcleo de las investigaciones llevadas a cabo en el seno del Instituto se ha orientado hacia un tema del que también he tenido la oportunidad de haber sido pionero en su estudio en España, habiendo dedicado al tema varios trabajos ${ }^{35}$ teóricos ${ }^{36}$, panorámicos ${ }^{37}$ y

35 A los que hace referencia, entre otros, Fernando Durán López, en Catálogo comentado de la autobiografía española (siglos XVII y XIX) (Madrid: Ollero \& Ramos Editores, 1997, 13 y 398-399).

36 Cf. José Romera Castillo: «La literatura autobiográfica como género literario», Revista de Investigación (C.U. de Soria) IV (1), (1980), 49-54; «La literatura, signo autobiográfico. El escritor, signo referencial de su escritura», en J. Romera Castillo (ed.), La literatura como signo (Madrid: Playor, 1981, 13-56); «Escritura y vida». Boletín de la Asociación de Profesores de Español 14, octubre (1993), 1 (Cuadernillo Cálamo, sección «Rúbrica»); «Literatura autobiográfica y docencia», en A. Rodríguez López-Vázquez (ed.), Simposio "Didáctica de Lenguas y Culturas" (La Coruña: Universidade, 1993, 11-28) -también en mi libro, Enseñanza de la Lengua y la Literatura (Madrid: UNED, 1996, 251-278) - y «Literatura y vida», en E. LópezBarajas Zayas (ed.), Las historias de vida y la investigación biográfica. Fundamentos y metodología (Madrid: UNED, 1996, 77-93).

37 Cf. J. Romera Castillo: «Panorama de la literatura autobiográfica en España (1975-1991)», Suplementos Anthropos 29 (1991), 170-184; «Literatura autobiográfica en España: Apuntes bibliográficos sobre los años ochenta», en A. Vilanova (ed.), Actas del X Congreso de la Asociación Internacional de Hispanistas (Barcelona: PPU, 1992, 
sobre algunos autores ${ }^{38}$. Me refiero a la escritura biográfica ${ }^{39}$ y autobiográfica, a las que dedicamos el VII y el II Seminarios Interna-

III, 241-248); «Escritos autobiográficos y teatro de la época (1916-1939)», en D. Dougherty y M. ${ }^{\mathrm{a}} \mathrm{F}$. Vilches de Frutos (eds.), El teatro en España entre la tradición y la vanguardia (1918-1939) (Madrid: CSIC/Fund. García Lorca/Tabacalera, 1992, 305-319); «Escritos autobiográficos de autores literarios traducidos en España (1990-92). Una selección)», Compás de Letras 1 (1992), 244-257; «Escritura autobiográfica cotidiana. El Diario en la literatura española actual (1975-1991)», Revista Marroquí de Estudios Hispánicos 3 (1994), 3-18; «Hacia un repertorio bibliográfico (selecto) de la escritura autobiográfica en España (1975-1992)», en J. Romera et alii (eds.), Escritura autobiográfica (Madrid: Visor Libros, 1993, 423-505); «Escritura autobiográfica en la España actual: Los pintores se retratan/los músicos se interpretan», en Romanistica Turkensis. Mélanges d'Etudes Romanes offerts à Lauri Lindgren à l'occasion de son 60e anniversaire (Turku: Turun Yliopisto, 1993, 207-220); «El descubrimiento del yo: pensadores y científicos se investigan a sí mismos», en J.M. ${ }^{2}$ Paz Gago et alii (eds.), Semiótica y Modernidad. Investigaciones Semióticas V (A Coruña: Universidade, 1994, I, 233-245); «Escritura autobiográfica de mujeres en España (1975-1991)», en J. Villegas (ed.), La mujer y su representación en las literaturas hispánicas (Actas del XI Congreso de la Asoc. Intern. de Hispanistas) (Irvine: University of California, 1994, II, 140-148); «Escritura autobiográfica hispanoamericana aparecida en España en los últimos años», en F. Carbó et alii (eds.), Homenatge a Amelia García-Valdecasas (Valencia: Universitat de València/Facultat de Filología, 1995, II, 727-740); «Senderos de vida en la escritura española (1993)», en M. Criado de Val (ed.), Actas del II Congreso Intern. sobre Caminería Hispánica (Guadalajara: Aache Ediciones, 1996, II, 461-478); «Senderos de vida en la escritura española (1995)", Boletín de la Unidad de Estudios Biográficos 1 (1996), 57-67; «Escritura autobiográfica», en J. Romera (ed.), El placer de leer (Un canon de lectura de la literatura actual), A Distancia (UNED), otoño (1997), 111-118; «Senderos de vida en la literatura española (1994)», en E. Ramón Trives y H. Provencio (eds.), Estudios de Lingüística Textual. Homenaje al Profesor Muñoz Cortés (Murcia: Universidad / CAM, 1998, 435-445) y «Perfiles autobiográficos de la "otra generación del 27" (la del humor)", en D. W. Flitter (ed.), Actas del XII Congreso de la AIH (Birmingham: Departament of Hispanic Studies: The University of Birmingham, 1998, IV, 241-247).

38 Cf. José Romera Castillo: «Tiempo de silencio' ¿un relato autobiográfico de ficción?», en VV. AA., 'Tiempo de silencio' de Luis Martín-Santos, 'Señas de identidad' de Juan Goytisolo. Deux romans de la rupture? (Toulouse: Université de Toulouse-Le Mirail, 1980, 15-29); «Autobiografía de Luis Cernuda: aspectos literarios», en L'Autobiographie en Espagne (Actes du II Colloque International) (Aix-En-Provence: Université de Provence, 1982, 279-294); «La memoria (auto)crítica del escritor incipiente Francisco Ayala», en A. Sánchez Trigueros y A. Chicharro Chamorro (eds.), Francisco Ayala teórico y crítico literario (Granada: Diputación Provincial, 1992, 6782); «Escritura autobiográfica de Miguel Delibes», en Cristóbal Cuevas García (ed.), Miguel Delibes. El escritor, la obra y el lector (Barcelona: Anthropos, 1992, 267-276); «Jirones autobiográficos y literarios de Carlos Edmundo de Ory. Epistolario a Ginés Liébana», en VV. AA., Scripta Philologica in honorem Juan M. Lope Blanch (México: UNAM, 1992, III, 441-453); «Polifonía literaria confesional de la España peregrina (Con un solo de Juan Gil-Albert)», en C. Simón y P. J. de la Peña (eds.), Homenaje a Juan GilAlbert (València: Consellería de Cultura de la Generalitat Valenciana, 1994, 53-74); «Junto a Juan Gil-Albert», El Mono-Gráfico (Valencia) 9 (1996), 65-74; «Edgar Neville y el cine (algunos testimonios)», «Cuadernos de Cultura» de A Distancia (UNED), otoño (1997), IX-XIII y «Apuntes sobre la actividad escénica madrileña (1919-1920) de García Lorca en su epistolario», en P. Guerrero Ruiz (ed.), Federico García Lorca en el espejo del tiempo (Alicante: Aguaclara / Caja de Ahorros del Mediterráneo, 1998, 193-201).

39 Cf. José Romera Castillo, «Ante las biografías literarias» y «Unas biografías de escritores españoles actuales», en José Romera Castillo y Francisco Gutiérrez Carbajo (eds.), Biografias literarias (1975-1997) (Madrid: Visor Libros, 1998, 11-25 y 243279 , respectivamente). 
cionales de nuestro Instituto, respectivamente, como hemos visto con anterioridad. Hasta el momento se han realizado dos tesis de doctorado: La literatura como conocimiento y participación en María Zambrano, de María Luisa Maillard García (1994) -publicada primero en microforma por la UNED, 1995 y posteriormente como María Zambrano. La literatura como conocimiento y participación (Lleida: Edicions de la Universitat, Ensayos / Scriptura n. $\left.{ }^{\circ} 6,1997\right)$ y Autobiografía y ficción en la novela española actual: J. Semprún, C. Barral, L. Goytisolo, Enriqueta Antolín y A. Muñoz Molina, de Alicia Molero de la Iglesia (1999) —que será publicada en microforma por la UNED-.

Asimismo se han sido realizado varias Memorias de Investigación: Parcelas autobiagráficas en la obra de Gabriel Miró, de Francisco Reus Boyd-Swan (1983); Lo autobiográfico en "La realidad y el deseo", de Luis Cernuda, de María del Mar Pastor Navarro (1985); La prosa autobiográfica de Carlos Barral, de Cecilio Díaz González (1991); Autobiografía y novela en algunas escritoras de la generación del 68, de Salustiano Martín González (1992); La escritura autobiográfica de Ramón Carnicer, de Helena Fidalgo Robleda (1994) y Los elementos autobiográficos en la narrativa de Fernando del Paso, de Alfredo Cerda Muños (1995).

\subsubsection{Literatura española del Siglo de Oro}

Otro eje de mis investigaciones ha sido el estudio de la literatura española de la época áurea, desde la perspectiva semiótica ${ }^{40}$. Como

40 Cf. José Romera Castillo, Notas a tres obras de Lope, Tirso y Calderón (Madrid: UNED, 1981) — con reseñas de Cristina González, «Romera Castillo, José: Estudios sobre «El Conde Lucanor» y Notas a tres obras de Lope, Tirso y Calderón», Ínsula 431 (1982), 9 y Peter W. Evans, en Bulletin of Hispanic Studies LX (1983), 340-341 (en inglés)-; La poesía de Hernando de Acuña (Madrid: Fundación Juan March, 1982; Serie Universitaria, n. ${ }^{\circ}$ 179); En torno a «El Patrañuelo» (Madrid: UNED, 1983); Frutos del mejor árbol. Estudios sobre teatro español del Siglo de Oro (Madrid: UNED, 1993) — con reseñas de Agustina Torres Lara, Epos X (1994), 589-590 y Francisco Gutiérrez Carbajo, «Nuevas investigaciones sobre el teatro clásico español», Cuadernos Hispanoamericanos 541-542 (1994), 243-247-; Calas en la literatura española del Siglo de Oro (Madrid: UNED, 1998); así como las ediciones de Joan Timoneda, El Patrañuelo (Madrid: Cátedra, 1986, 2. ed.; Letras Hispánicas, n. ${ }^{\circ}$ 94) - con reseñas de Evangelina Rodríguez Cuadros, «Joan Timoneda como mediador literario», Ínsula 392-393 (1979), 12; Émile Amaud, Caravelle. Cahiers du Monde 
consecuencia de ello, se han realizado dos tesis de doctorado: La poesía pastoril española del siglo XVI, de Ramón Mateo Mateo (1990) y El manuscrito 570 de la Biblioteca Real y la obra de Damasio de Frías, de Carmen Ponz Guillén (1990) — publicada en microforma por la UNED - Así como las Memorias de Investigación: Las imágenes de la luz en el "Libro de la Vida» de Santa Teresa de Jesús, de Monserrat Izquierdo Sorlí (1983) y Propuesta para una lectura de las Cartas de Pedro de Valdivia, de Jimena Sepúlveda Brito (1992).

\subsubsection{Literatura moderna y contemporánea en español}

Sobre este periodo, siguiendo la senda de mis numerosos trabajos ${ }^{41}$, se han llevado a cabo seis tesis de doctorado: La última producción literaria de Camilo José Cela (1983-1988), de Dieudonné Mendogo Minsongui (1990); La novelística de Juan Madrid, de Nzachée Noumbissi (1993); Hacia una caracterización de la escritura femenina. La narrativa de Luisa Josefina Hernández, escritora mexicana, de Ra-

\footnotetext{
Hispanique et Luso-Brésilien 34 (1980), 286-288; F. William Forbes, Hispania 63. 4 (1980), 774-775 (en inglés) y Rafael Ferreres, «Sobre una crítica de mi edición de $E l$ Patrañuelo", Ínsula 411 (1981), 14-; El segundo David (Auto sacramental supuesto e inédito atribuido a Calderón), Suplemento de la revista Número (Madrid), 2 (mayojunio, 1982) - por el ms. autógrafo, encontrado después, se supo que era de Lope de Vega-; Pedro Calderón de la Barca, Casa con dos puertas, mala es de guardar y El galán fantasma (Barcelona: Plaza \& Janés, 1984; Biblioteca Crítica de Autores Españoles, n. ${ }^{\circ}$ 19) y Gonzalo Argote de Molina, El «Discurso sobre la Poesía Castellana» (Madrid: Visor Libros, 1995; edición facsímil de la de Eleuterio F. Tiscornia; Colección Biblioteca Filológica Hispana, n. ${ }^{\circ} 6$ ).

41 Cf. los estudios de orientación semiótica de José Romera Castillo, Con Antonio Gala (Estudios sobre su obra) (Madrid: UNED, 1996, Aula Abierta n. ${ }^{\circ} 100$; con «Pórtico» de Antonio Gala) - reseñado anónimamente, A Distancia, primavera (1997), 35; Ana Padilla Mangas, Signa 6 (1997), 429-434; José Manuel Reyes (Rutgers University), Gestos (University of California, Irvine) 24 (1997), 202-203; Emilia Cortés Ibáñez, Epos XIII (1997), 536-539; Alfredo Rodríguez López-Vázquez, Lenguaje y Textos 10 (1997), 373-374 y Coral López Gómez, España Contemporánea. Revista de Literatura y Cultura X.2 (1997), 99-100-; las ediciones de las obras de Antonio Gala, Los verdes campos del Edén y El cementerio de los pájaros (Barcelona: Plaza \& Janés, 1986; Biblioteca Crítica de Autores Españoles, n. ${ }^{\circ}$ 52) - con reseñas de Dinda L. Gorlée, Epos IV (1988), 485-487 [también, «Dos textos de A. Gala: Juntos, pero no revueltos», Ínsula 505 (1989), 26] y Phyllis Zatlin Boring, Gestos 5 (1988), 166-167 (en inglés)-; Carmen Carmen (Madrid: Espasa-Calpe, 1988; Colección Austral, n. ${ }^{\circ}$ 65); Cristóbal Colón (Madrid: Espasa-Calpe, 1990; Colección Austral, n. ${ }^{\circ}$ 138); además de numerosísimos trabajos sobre otros autores y obras de este periodo diseminados en volúmenes colectivos y artículos de revistas que, por su extensión, no puedo pormenorizar aquí.
} 
quel Gutiérrez Estupiñán (1995) —publicada en microforma por la UNED en 1996-; Sofía Casanova: mito y literatura, de M. ${ }^{a}$ Rosario Martínez Martínez (1996); Aproximación semiótica al teatro histórico de Domingo Miras y Aimé Césaire, de André Mah (1997) y Presencia de América en la novelística de Camilo José Cela, Miguel Delibes y Gonzalo Torrente Ballester, de Gloria Inés Sanabria Martínez (1997).

Así como varias Memorias de Investigación: Estudio semiótico de "Rosalía", de Pérez Galdós, de Jesús Torrecilla Cabañas (1986); El cuento en "El País" (1976-1980), de Felipe Díaz Pardo (1991); Hacia un análisis pragmático del discurso teatral (Teoría y praxis: "Ulf" de Juan Carlos Gené), de Graciela Frega (1991); Reflexiones sobre la lectura (para)literaria del suceso, de Laura Serrano de Santos (1992); La novela histórica en España durante la última década (1980-1991), de Alfredo Caunedo Álvarez (1992) y Presencia de la poesía española del siglo XX en Italia: las traducciones a partir de 1975, de Coral García Rodríguez (1996).

\subsubsection{Literatura y nuevas tecnologías}

Finalmente en este novedoso ámbito, del que también he sido pionero en España y al que dedicamos el VI Seminario de nuestro Instituto $^{42}$, al estudiar el proceso de semiosis que la literatura adquiere a través de estas nuevas herramientas, en la línea de mis trabajos ${ }^{43}$, se ha realizado la Memoria de Investigación, Hipertexto y literatura, de

42 Cf. José Romera Castillo, Francisco Gutiérrez Carbajo y Mario García-Page (eds.), Literatura y multimedia (Madrid: Visor Libros, 1997).

${ }_{43}$ Cf. José Romera Castillo, «Literatura y multimedia: una apuesta por el ciberespacio didáctico», en mi libro, Enseñanza de la Lengua y la Literatura (Propuestas metodológicas y bibliográficas) (Madrid: UNED, 1996, 217-250); «Presentación: Prepararse para el futuro (in)mediato» y «Literatura y nuevas tecnologías», en José Romera Castillo et alii (eds.), Literatura y multimedia (Madrid: Visor Libros, 1997, 79 y 13-82, respectivamente); «Literatura y nuevas tecnologías», en Catalina M. Alonso y Domingo J. Gallego (eds.), La informática desde la perspectiva de los educadores (Madrid: R. A. Comunicación Gráfica, 1997, t. II, 721-726; en soporte impreso y CDROM); «Literatura y nuevas tecnologías», Cuadernos Cervantes de la Lengua Española 18 (1998), 77-83; «Los multimedia ¿un nuevo mito para la literatura?», en Túa Blesa (ed.), Mitos (Actas del VII Congreso Internacional de la Asociación Española de Semiótica) (Zaragoza: Anexos de Tropelías, 1998, I, 207-212) y «Sobre literatura y multimedia», en Nicole Delbecque y Christian De Paepe (eds.), Estudios en honor del profesor Josse de Kock (Lovaina, Bélgica: Leuven University Press, 1998, 919-924). 
EL INSTITUTO DE SEMIÓTICA LITERARIA, TEATRAL Y NUEVAS TECNOLOGIAS...

Beatriz Paternain Miranda (1997), que pronto se convertirá en tesis de doctorado.

\subsection{Cursos}

Para promover el estudio de la literatura, el teatro, las nuevas tecnologías, dentro del ámbito de la semiótica, el Instituto viene organizando, a lo largo de sus años de existencia, varias modalidades de cursos:

\subsubsection{Cursos de Tercer Ciclo}

Los estudios de este ciclo culminan la formación universitaria, a través de los cursos de doctorado, con el fin de iniciar y promover la investigación. Dentro del Departamento de Literatura Española y Teoría de la Literatura, de la Facultad de Filología de la UNED, desde que se iniciasen, bajo mi coordinación general, he impartido varios cursos de doctorado: «La novela española de posguerra» (1980-81 y 1981-82); «Poesía italianizante del Renacimiento español» (1982-83 y 1983-84); «El petrarquismo en la lírica española del siglo XVI» (198485 hasta 1986-87); «Literatura española del Siglo de Oro y semiótica» (1987-88 hasta 1992-93) y «Lo autobiográfico en la literatura española actual» (1987-88 hasta 1998-99). Gracias a los cuales diferentes alumnos han realizado Memorias de Investigación y tesis de doctorado, como hemos visto anteriormente.

Asimismo, he impartido cursos de doctorado y de postgrado, desde la óptica semiótica, tanto en otras Universidades españolas: sobre $L a$ Celestina (Universidad de Oviedo, 1989) — «El diálogo en La Celestina»- y «Narratología» (Universidad de Almería, 1995), como en Universidades del extranjero: «Teatro español del Siglo de Oro» (Universidad Autónoma de México, 1982), «Semiótica y Literatura» (Universidad Nacional de Tucumán, Argentina, 1987) y «Literatura y semiótica» (Instituto de Investigaciones Filológicas, UNAM, México, 1990). 


\subsubsection{Cursos de extensión universitaria}

La UNED, a través de los estudios de Enseñanzas no Regladas, tiene varios programas de enseñanza, con el fin de complementar y reciclar a profesores y alumnos universitarios de diferentes especialidades. Dentro del Programa de Enseñanza Abierta, desde 1989, vengo dirigiendo anualmente un curso, que dura desde noviembre a junio, sobre Literatura y Semiótica: Método y práctica ${ }^{44}$. Así como en el Programa de Formación del Profesorado, desde 1984, dirijo otro curso, cada año, desde noviembre a junio, sobre Enseñanza de la Lengua y la Literatura $^{45}$, donde figuran aspectos del estudio y análisis de la literatura y el teatro, desde una perspectiva semiótica. A lo largo de los años, han cursado los mismos cerca de cuatro mil alumnos, la mayoría de ellos profesores de Primaria, Secundaria y Bachillerato - algunos también de Universidad-, así como alumnos universitarios que deseaban completar su formación con facetas, referidas a los temas propuestos, que no habían sido atendidas o lo habían hecho sin mucha profundización en sus curricula.

\subsubsection{Cursos de corta duración}

Asimismo, dentro de esta misma línea, he dirigido varios cursos de verano o de otro tipo, diseñados para los destinatarios anteriormente reseñados y con una duración media de cinco días, en los que han impartido docencia destacados especialistas y en los que he intervenido siempre con alguna exposición. Hasta el momento, se han llevado a cabo veinticinco: Literatura y periodismo, en los I Cursos de Verano de la UNED, con las intervenciones de Luis Antonio de Villena, Antonio Carvajal y Antonio Hernández, entre otros (Elche, 11-14 de julio, 1989); La literatura española en la actualidad (1975-1991), en

44 Como puede comprobarse en el manual de estudio del curso de José Romera Castillo, Literatura, teatro y semiótica: Método, prácticas y bibliografía (Madrid: UNED, 1998).

45 Como puede comprobarse en el manual de estudio del curso de José Romera Castillo, Enseñanza de la Lengua y la Literatura (Propuestas metodológicas y bibliográficas) (Madrid: UNED, 1996; 1. ${ }^{\text {a }}$ reimpresión en julio de 1998). Con reseñas de José Manuel Corriente Cordero, Signa 6 (1997), $435-437$ y reseña anónima, A Distancia (UNED), primavera (1997), 34-35. 
los II Cursos de Verano de la UNED, con las intervenciones de Antonio Gala, Soledad Puértolas, Rafael Pérez Sierra, M. ${ }^{a}$ Carmen Bobes, entre otros (Ávila, 15-19 de julio, 1991); 500 años de Lengua Española, con las intervenciones de Antonio Quilis, Miguel Ángel Pérez Priego, Francisco Marsá y M. ${ }^{a}$ Luz Gutiérrez (Melilla, 4-8 de mayo, 1992); El teatro español actual. El teatro de Antonio Gala, en los III Cursos de Verano de la UNED, con las intervenciones de Antonio Gala, Andrés Amorós, César Oliva, Luciano García Lorenzo, José María Díez Borque, Evangelina Rodríguez Cuadros, entre otros (Denia, 20-24 de julio, 1992); Tertulias Literarias, un ciclo con escritores en el XX Aniversario de la creación de la UNED, organizado por la Facultad de Filología, con las intervenciones de Alfredo Bryce Echenique, Francisco Brines, Abelardo Linares y Ángel Lerchundi (Madrid y varios Centros Asociados a la UNED: Valdepeñas, Sevilla y Portugalete, marzo y abril, 1993); La novela española actual (19751992), en los IV Cursos de Verano de la UNED, con las intervenciones de Fernando Sánchez Dragó, Ángel Basanta, Joaquín Marco, Fanny Rubio, Julio Rodríguez Puértolas, Gregorio Torres Nebrera, entre otros (Ávila, 19-23 de julio, 1993); Literatura y autobiografía, en el Centro Asociado a la UNED de Cádiz, con las intervenciones de Manuel Ramos Ortega, Francisco Gutiérrez Carbajo, José Pallarés, etc. (Cádiz, 8-11 de marzo, 1994); Teatro: texto y representación, en los Cursos de Invierno de la UNED-Fundación Cultural Santa Teresa, con las intervenciones de Miguel Á. Pérez Priego, José Mà . Díez Borque, M. ${ }^{a}$ Francisca Vilches de Frutos, Luciano García Lorenzo, entre otros (Ávila, 11-13 de marzo, 1994); La poesía española actual (1975-1994), en los V Cursos de Verano de la UNED, con las intervenciones de Jaime Siles, Luis García Montero, Antonio Hernández, Jaume Pont, José M. ${ }^{a}$ Balcells, Mario Hernández, entre otros (Ávila, 18-22 de julio, 1994); Técnicas de expresión escrita, en el centro Asociado a la UNED de Melilla, con las intervenciones de Miguel Á. Pérez Priego, Vidal Lamíquiz y M. ${ }^{a}$ Luz Gutiérrez (Melilla, 25-28 de abril, 1995); Análisis e interpretación del texto literario, en el Centro Asociado a la UNED de Valdepeñas (3-7 de julio, 1995); Literatura y medios de comunicación social, en los VI Cursos de Verano de la UNED, con las intervenciones de Luis Antonio de Villena, Javier Lostalé, Andrés Trapiello, Agustín Remesal, Juan Cruz, Miguel GarcíaPosada, Rosa Navarro, Agustín Cerezales y Luis de la Peña (Ávila, 1721 de julio, 1995); Literatura y medios de comunicación, en el Centro Asociado a la UNED de Melilla, con las intervenciones de Agustín Remesal, Javier Lostalé, José Esteban, Felipe Benítez Reyes y José M. 
Caballero Bonald (Melilla, 16-20 de octubre, 1995); Jornadas sobre Literatura Española Actual (XII Ciclo de Poesía y VIII Ciclo de Narrativa), organizadas por la Delegación Provincial del Ministerio de Cultura, el Centro Asociado a la UNED y la Consejería de Cultura, con las intervenciones de Fanny Rubio, Rafael Pérez Estrada, César Simón, Clara Sánchez, Luis García Montero, Almudena Grandes y José L. Fernández de la Torre (Melilla, 13-15 de diciembre, 1995); De la literatura al cine (en colaboración con F. Gutiérrez Carbajo), en los VII Cursos de Verano de la UNED, con las intervenciones de Gonzalo Suárez, Andrés Amorós, Román Gubern, Eduardo Mendicutti, José Luis Alonso de Santos, etc. (Ávila, 8-12 de julio, 1996); Escritura femenina, organizado por la Delegación Provincial del Ministerio de Educación y Cultura, la Ciudad Autónoma y el Centro Asociado a la UNED, con las intervenciones de Clara Sánchez, Clara Janés, Marina Mayoral, Carme Riera, Ana Rosetti y Lourdes Ortiz (Melilla, 14-18 octubre, 1996); Lengua, culturas e identidad, en el Centro Asociado a la UNED, con las intervenciones de José María Merino, Miguel Á. Pérez Priego, Humberto López Morales, M. ${ }^{\mathrm{a}}$ Luz Gutiérrez, etc. (Melilla 5-9 de mayo, 1997); Narrativa española actual, en el Centro Asociado a la UNED de Jaén-Úbeda, con las intervenciones de José M. Caballero Bonald, M. ${ }^{\mathrm{a}}$ Carmen Bobes, etc. (Úbeda, 9-10 y 16-17 de mayo, 1997); Análisis de la literatura política y fantástica, en el Centro Asociado a la UNED (Valdepeñas, 23-27 de junio, 1997); Las humanidades y las nuevas tecnologías, curso institucional en los VIII Cursos de Verano de la UNED, con las intervenciones de Ángel López García, Francisco A. Marcos Marín, Manuel Alvar Ezquerra, José Antonio Millán, Valerio Báez San José, etc. (Ávila, 7-11 de julio, 1997); Escritores de hoy, organizado por la Dirección General del Libro, Archivos y Bibliotecas, el Centro Asociado a la UNED y la Consejería de Cultura, con las intervenciones de Alfredo Bryce Echenique, Eduardo Mendicutti, Almudena Grandes, Arturo PérezReverte, Luis Alberto de Cuenca, Juan Eslava Galán, Laura Freixas y José Esteban (Melilla, 20-23 de octubre, 1997); Escorzo sobre Federico García Lorca, con motivo del centenario del nacimiento del poeta, en el Centro Asociado a la UNED de Melilla, con las intervenciones de Juan Carlos Rodríguez, Francisco Abad, M. ${ }^{a}$ Clementa Millán, Francisco Díaz de Castro, etc. (Melilla, 12-15 de mayo, 1998); A cien años de la generación del 98, en el Centro Asociado a la UNED de Jaén, con las intervenciones de Manuel Alvar, Andrés Trapiello, Pedro Cerezo Galán, Juan A. Lacomba, etc. (Úbeda, 22-24 de mayo, 1998); El 98, cien años después, curso institucional en los IX Cursos 
de Verano de la UNED, con las intervenciones de Santos Juliá, Javier Tusell, Miguel Á. Pérez Priego, etc. (Ávila, 20-24 de julio, 1998) y Jóvenes escritores (Ante el final del milenio), organizado por la Dirección General del Libro, Archivos y Bibliotecas del Ministerio de Educación y Cultura/Consejería de Cultura de la Ciudad Autónoma / Centro de la UNED, con las intervenciones de Andrés Neuman Galán, Leopoldo Alas, Martín Casariego, Nicolás Casariego y Basilio Rodríguez (Melilla, 19-23 de octubre, 1998).

\section{FINAL}

De lo expuesto anteriormente, se puede corrobar la importante labor de investigación y difusión llevada a cabo en el seno de nuestro Instituto de Semiótica Literaria, Teatral y Nuevas Tecnologías, que unida a la realizada por otros investigadores - como he tenido la oportunidad de ocuparme en diversos estados de la cuestión y como se consigna en este número monográfico- da muestra de un granado y maduro quehacer dentro de los estudios semióticos en España. 